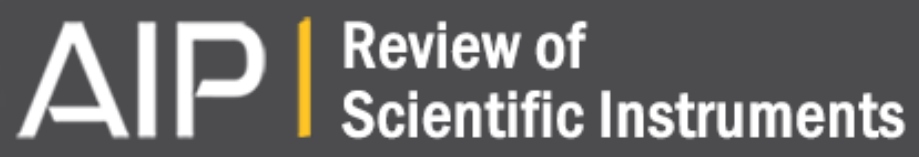

Spatially uniform calibration of a liquid xenon detector at low energies using $K 83 \mathbf{m ~ r}$

A. Manalaysay, T. Marrodán Undagoitia, A. Askin, L. Baudis, A. Behrens, A. D. Ferella, A. Kish, O. Lebeda, R.

Santorelli, D. Vénos, and A. Vollhardt

Citation: Review of Scientific Instruments 81, 073303 (2010); doi: 10.1063/1.3436636

View online: http://dx.doi.org/10.1063/1.3436636

View Table of Contents: http://scitation.aip.org/content/aip/journal/rsi/81/7?ver=pdfcov

Published by the AIP Publishing

\section{Articles you may be interested in}

Cryogenic Technology Development For The MEG Liquid Xenon Calorimeter

AIP Conf. Proc. 981, 366 (2008); 10.1063/1.2898990

Low energy neutrino astrophysics with the large liquid-scintillator detector LENA

AIP Conf. Proc. 944, 82 (2007); 10.1063/1.2818554

DEAP \& CLEAN Detectors for Low Energy Particle Astrophysics

AIP Conf. Proc. 870, 205 (2006); 10.1063/1.2402618

Absolute calibration of a multichannel plate detector for low energy $\mathrm{O}, \mathrm{O}-$, and $\mathrm{O}+$

Rev. Sci. Instrum. 71, 1355 (2000); 10.1063/1.1150462

Calibration of the Galileo microchannel plates with the Xe 2+-Xe 13+ and C 2+-C 6+ ions in the energy range from 0.5 to $150 \mathrm{keV/q}$ (abstract)

Rev. Sci. Instrum. 71, 1100 (2000); 10.1063/1.1150146

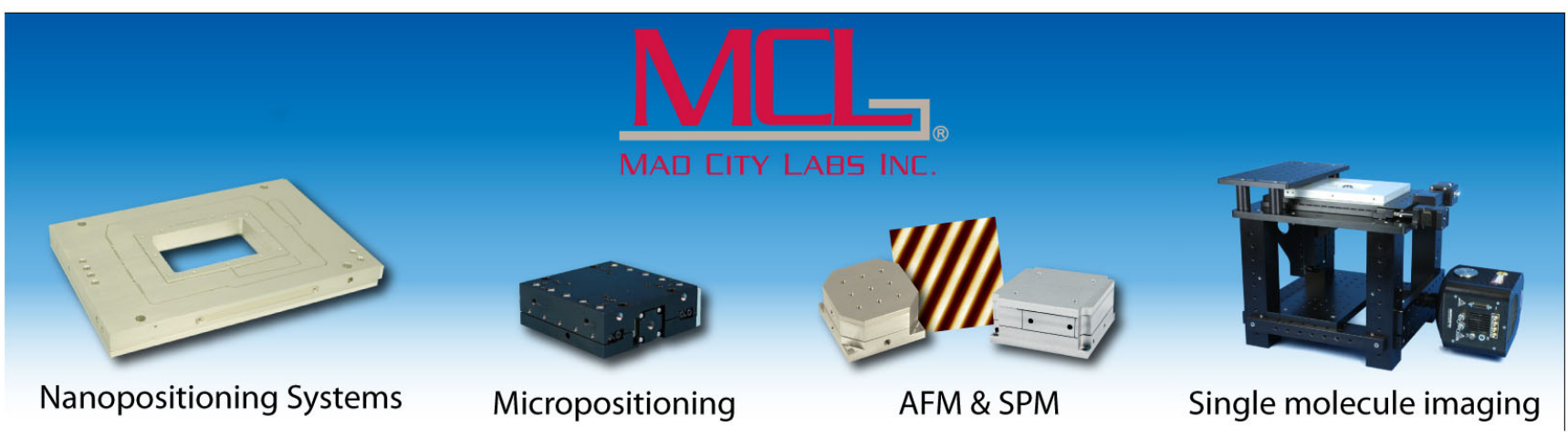




\title{
Spatially uniform calibration of a liquid xenon detector at low energies using ${ }^{83 \mathrm{~m}} \mathrm{Kr}$
}

\author{
A. Manalaysay, ${ }^{1,2, a)}$ T. Marrodán Undagoitia, ${ }^{1}$ A. Askin, ${ }^{1}$ L. Baudis, ${ }^{1}$ A. Behrens, ${ }^{1}$ \\ A. D. Ferella, ${ }^{1}$ A. Kish, ${ }^{1}$ O. Lebeda,${ }^{3}$ R. Santorelli, ${ }^{1}$ D. Vénos, ${ }^{3}$ and A. Vollhardt ${ }^{1}$ \\ ${ }^{1}$ Physik-Institut, Universität Zürich, Zürich 8057, Switzerland \\ ${ }^{2}$ Department of Physics, University of Florida, Gainesville, Florida 32611, USA \\ ${ }^{3}$ Nuclear Physics Institute, Academy of Sciences of the Czech Republic, CZ 25068 Řež near Prague, \\ Czech Republic
}

(Received 3 December 2009; accepted 1 May 2010; published online 14 July 2010)

\begin{abstract}
A difficult task with many particle detectors focusing on interactions below $\sim 100 \mathrm{keV}$ is to perform a calibration in the appropriate energy range that adequately probes all regions of the detector. Because detector response can vary greatly in various locations within the device, a spatially uniform calibration is important. We present a new method for calibration of liquid xenon (LXe) detectors, using the short-lived ${ }^{83 \mathrm{~m}} \mathrm{Kr}$. This source has transitions at 9.4 and $32.1 \mathrm{keV}$, and as a noble gas like $\mathrm{Xe}$, it disperses uniformly in all regions of the detector. Even for low source activities, the existence of the two transitions provides a method of identifying the decays that is free of background. We find that at decreasing energies, the LXe light yield increases, while the amount of electric field quenching is diminished. Additionally, we show that if any long-lived radioactive backgrounds are introduced by this method, they will present less than $67 \times 10^{-6}$ events $\mathrm{kg}^{-1}$ day $^{-1} \mathrm{keV}^{-1}$ in the next generation of LXe dark matter direct detection searches. () 2010 American Institute of Physics. [doi:10.1063/1.3436636]
\end{abstract}

\section{INTRODUCTION}

Liquid xenon (LXe) particle detectors are commonly used in the fields of dark matter direct detection, ${ }^{1-5}$ neutrinoless double-beta decay searches, ${ }^{6}$ collider experiments, ${ }^{7}$ and in nuclear medicine. ${ }^{8,9}$ In these applications, energy deposition is measured by counting scintillation photons, ionization, or both. The high electron mobility ${ }^{10}$ and scintillation properties $^{11}$ of this liquid contribute to its versatility. LXe response depends on the electronic stopping power $(d E / d x)$ to the incoming particle, which itself depends on both the identity and energy of the particle. It is therefore necessary to calibrate a detector's energy scale with a source whose response is known relative to the particles under study. One common such "reference source" is ${ }^{57} \mathrm{Co}$, which emits $\gamma$ rays predominantly at $122 \mathrm{keV}$.

Dark matter direct detection experiments search for low energy nuclear recoils caused by the scattering of weakly interacting massive particles (WIMPs) with atomic nuclei. ${ }^{12-14}$ There are two main problems involved in using ${ }^{57} \mathrm{Co}$ to calibrate LXe detectors for this application. The first is that the $\gamma$-ray energy is much higher than the recoiling nuclei energy produced by WIMP interactions. Second, the attenuation length of $122 \mathrm{keV} \gamma$ rays in LXe is $\sim 2.5 \mathrm{~mm}$, and hence the energy deposition will be highly localized as compared with the tens of centimeter typically characterizing the size of such detectors. The two problems are actually compounded because the attenuation length of $\gamma$ rays decreases as their energy decreases, and therefore sources pro-

\footnotetext{
${ }^{\text {a) }}$ Author to whom correspondence should be addressed. Electronic mail: aaronm@physik.uzh.ch.
}

viding lower-energy $\gamma$ rays will give an even more localized response than ${ }^{57} \mathrm{Co}$. The topic of localization is not only an issue for point sources placed outside the detector but also for point sources placed inside the detector. In the latter case, the source must be attached to a mounting device of some kind; for low energy $\gamma$-ray sources, any device used for this purpose will likely block some of the scintillation light and potentially distort any existing applied electric fields. It is therefore not possible to calibrate a detector with an internal point source under the same conditions that would exist during the actual measurement. To avoid these difficulties, short-lived noble gas sources can be used which diffuse uniformly in LXe. The XENON10 experiment used the metastable ${ }^{131 \mathrm{~m}}$ Xe. ${ }^{4,15}$ This source solves the second problem (spatial localization), but its $164 \mathrm{keV}$ transition does not overcome the problem of an appropriate energy scale. Additionally, due to its half-life of 12 days, the detector must sit for approximately 2.5 months following a calibration until the source activity has dropped to $1 \%$ of its initial value.

A promising alternative solution is to use the metastable ${ }^{83 \mathrm{~m}} \mathrm{Kr}$, first proposed in Ref. 16. This source has been used for calibrations of detectors in the large electron-positron collider, ${ }^{17,18}$ as well as in the KATRIN experiment which attempts to measure the electron neutrino absolute mass. ${ }^{19}$ ${ }^{83 \mathrm{~m}} \mathrm{Kr}$ should diffuse uniformly in a LXe detector, addressing the issue of spatial uniformity (while perfect uniformity of deposition is not explicitly required, a uniform deposition allows for calibration of the full detector in the minimum time). Additionally, its two de-excitation lines at 9.4 and 32.1 $\mathrm{keV}$ lie in the energy range of interest for dark matter direct searches, and its half-life of only $1.8 \mathrm{~h}$ allows for a short 


\begin{tabular}{|c|c|c|}
\hline $\begin{array}{l}32.1 \mathrm{keV} \\
(1.83 \mathrm{~h})\end{array}$ & $\begin{array}{l}76 \% \\
9 \% \\
15 \%\end{array}$ & $\begin{array}{l}\mathrm{IC}(30 \mathrm{keV})+\mathrm{A}(2 \mathrm{keV}) \\
\mathrm{IC}(18 \mathrm{keV})+\mathrm{A}(10 \mathrm{keV})+2 \times \mathrm{A}(2 \mathrm{keV}) \\
\mathrm{IC}(18 \mathrm{keV})+\mathrm{X}(12 \mathrm{keV})+\mathrm{A}(2 \mathrm{keV})\end{array}$ \\
\hline $\begin{array}{l}9.4 \mathrm{keV} \\
(154 \mathrm{~ns})\end{array}$ & $\begin{array}{l}95 \% \\
5 \%\end{array}$ & $\begin{array}{l}\mathrm{IC}(7.6 \mathrm{keV})+\mathrm{A}(1.8 \mathrm{keV}) \\
\gamma(9.4 \mathrm{keV})\end{array}$ \\
\hline
\end{tabular}

FIG. 1. The decay scheme and branching ratios of ${ }^{83 \mathrm{~m}} \mathrm{Kr}$. The decay always passes through two transitions, giving mostly internal conversion (IC) and Auger (A) electrons. A small amount of the energy is carried by gamma $(\gamma)$ and $\mathrm{x}$ rays $(\mathrm{X})($ Ref. 18).

turnaround time following measurement. Simultaneous with the work presented here, another group has performed a demonstration of a similar ${ }^{83 \mathrm{~m}} \mathrm{Kr}$ introduction method in a single-phase LXe chamber. ${ }^{20}$ Here we present a successful implementation of this calibration source in a small dualphase LXe prototype detector. Furthermore, we show results of measurements of the LXe energy scale linearity, evolution of energy resolution with energy, effects of LXe response under applied electric fields, and set limits on the level of long-lived radiocontaminants introduced by this method.

\section{EXPERIMENTAL METHODS}

\section{A. The ${ }^{83 \mathrm{~m}} \mathrm{Kr}$ source}

${ }^{83 \mathrm{~m}} \mathrm{Kr}$ is produced by the decay of ${ }^{83} \mathrm{Rb}$ via pure electron capture. Following this process, the nucleus is left in any of ${ }^{83} \mathrm{Kr}$ 's many excited states lying below the $Q$ value of the $\mathrm{Rb}$ decay $(910 \mathrm{keV})$. Regardless of the initial krypton excited state, the nucleus rapidly de-excites within picoseconds to the isomeric state ${ }^{83 \mathrm{~m}} \mathrm{Kr}$, located $41.5 \mathrm{keV}$ above the ground state. Isomeric krypton decays with a half-life of $1.83 \mathrm{~h}$ to the first ${ }^{83} \mathrm{Kr}$ excited state $(9.4 \mathrm{keV})$, which then decays to the ground state with a half-life of $154 \mathrm{~ns}^{21}$ The decay scheme of ${ }^{83 \mathrm{~m}} \mathrm{Kr}$ is shown in Fig. 1, indicating that most of the released energy is carried by internal conversion and $\mathrm{Au}-$ ger electrons. ${ }^{18}$ The $6 \mathrm{kBq}{ }^{83} \mathrm{Rb}$ source used in this study was produced at the Nuclear Physics Institute, Řež (Czech Republic). This institute also provides ${ }^{83} \mathrm{Rb}$ for the KATRIN experiment. ${ }^{19}$ The parent ${ }^{83} \mathrm{Rb}$ is produced in the U-120M cyclotron from the reaction ${ }^{\text {nat }} \mathrm{Kr}(p, x n){ }^{83} \mathrm{Rb}$ by irradiating a medium-pressure krypton target with $27 \mathrm{MeV}$ protons. The product, deposited on the target chamber walls, is then washed into several tens of milliliters of high purity water $(<0.07 \mu \mathrm{S} / \mathrm{cm})$. An appropriate amount of the target eluate is then absorbed in zeolite beads ( $2 \mathrm{~mm}$ diameter, Merck), which acts as a molecular sieve. Zeolite was chosen due to its ability to allow for efficient emanation of ${ }^{83 \mathrm{~m}} \mathrm{Kr}$ in vacuum, while exhibiting high retention of the mother ${ }^{83} \mathrm{Rb}$ in its porous structure. For details on the production process, we refer to Ref. 22. In addition to ${ }^{83} \mathrm{Rb},{ }^{84} \mathrm{Rb}$ and ${ }^{86} \mathrm{Rb}$ isotopes are also produced; however, they decay to stable $\mathrm{Kr}$ isotopes and hence introduce no radioactive backgrounds. Since ${ }^{83} \mathrm{Rb}$ decays with a half-life of 86.2 days, the source strength decreased to $\sim 3 \mathrm{kBq}$ by the end of these measurements.

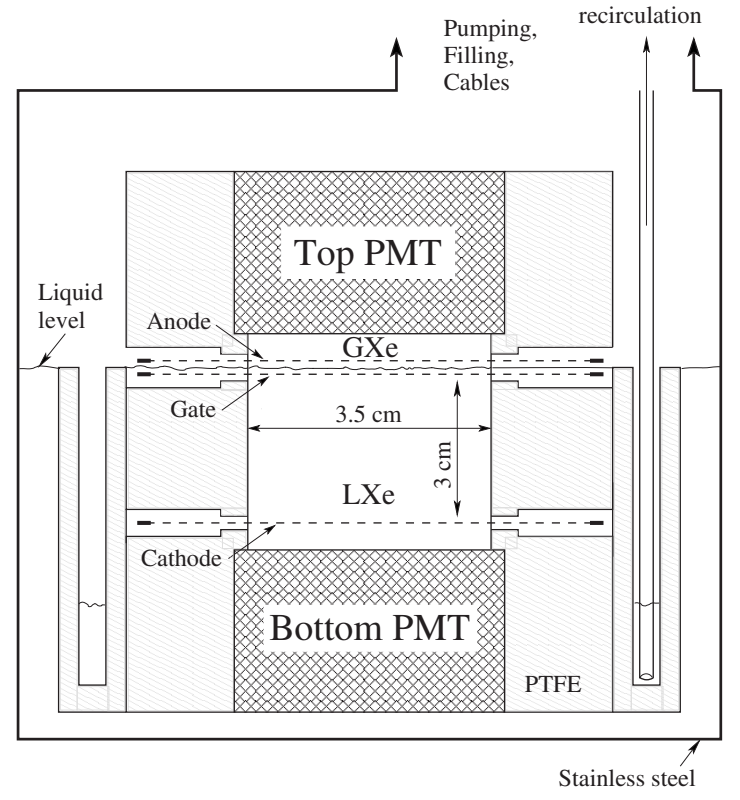

FIG. 2. Schematic of the dual-phase Xürich detector. The PTFE structure holds the PMTs and grid electrodes (see text), defining an active region 3.5 $\mathrm{cm}$ in diameter and $3 \mathrm{~cm}$ high.

\section{B. Detector system}

The "Xürich" detector, used for studying the ${ }^{83 \mathrm{~m}} \mathrm{Kr}$ decay, is shown schematically in Fig. 2. It is a dual-phase (liquid and gas) LXe time projection chamber (TPC). The system was built at the Universität Zürich specifically for testing LXe's response to low energy interactions. The stainless steel vessel in Fig. 2 is located inside a vacuum cryostat, with cooling provided via a copper cold finger immersed in liquid nitrogen. The temperature and pressure are held constant at $175 \mathrm{~K}$ and 1.8 bar, respectively, and the detector was operated stably for approximately 2.5 months. Xürich's cylindrical active region, $3.5 \mathrm{~cm}$ in diameter and $3 \mathrm{~cm}$ in height (80.8 $\mathrm{g}$ of LXe), is defined by a polytetrafluoroethylene (PTFE) cylinder on the perimeter and grid electrodes above (gate) and below (cathode). A third grid electrode (anode) is located above the gate grid, with the liquid level lying between the gate and anode grids. Two Hamamatsu R9869 (Ref. 23) photomultiplier tubes (PMTs) view the active volume, one from below and one from above. A total of $1.76 \mathrm{~kg}$ is used to fill the stainless steel vessel.

The cathode and gate grids apply an electric field of typically $\sim 1 \mathrm{kV} / \mathrm{cm}$ which is used to drift electrons away from an interaction site toward the gate grid. Once the electrons pass through the gate grid, they arrive at the liquid surface and are extracted to the gas by an electric field of $\sim 10 \mathrm{kV} / \mathrm{cm}$ that then accelerates the electrons through the gas until they collect on the anode grid. The high voltage applied to the grids is supplied by a CAEN model A1526 module. During their transit through the gas, the electrons collide with Xe atoms with sufficient energy to produce scintillation light. Therefore, the typical result of a particle interaction is a prompt scintillation signal ( $\mathrm{S} 1)$ emitted from the interaction site itself, followed by a delayed scintillation signal (S2) produced as the electrons travel through the gas under the influence of the extraction field. In this way, both 


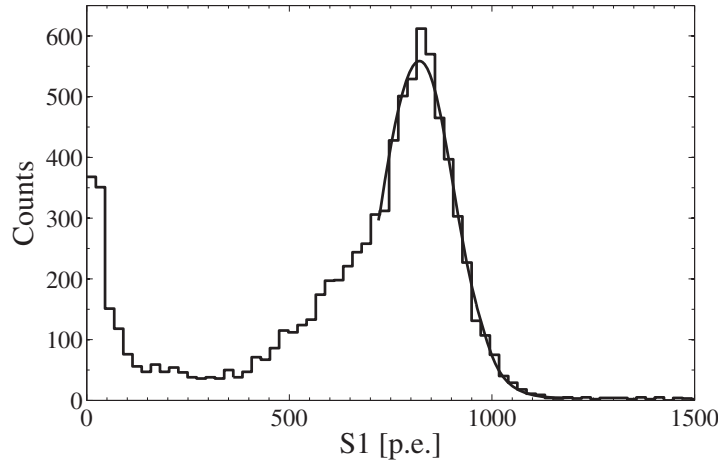

FIG. 3. Spectrum obtained from ${ }^{57} \mathrm{Co}$ at zero field and Gaussian fit. The run-averaged $\mathrm{LY}$ is $6.38 \mathrm{pe} / \mathrm{keV}$.

the scintillation and ionization signals are measured by the PMTs. This technique is used for charge readout because it provides superior amplification over more traditional methods. ${ }^{24,25}$ Additionally, the $z$ position of the event can be inferred by the delay time between the S1 and S2 signals since the electron drift velocity is well known as a function of applied field. ${ }^{10}$

The light yield (LY) is defined as the number of photoelectrons (pe) emitted from the PMT photocathodes per unit energy, and is customarily quoted based on the primary emission of ${ }^{57} \mathrm{Co}$. When Xürich is operated in single-phase mode with the liquid level above the top PMT, the ${ }^{57} \mathrm{Co}$ source produces $\sim 10 \mathrm{pe} / \mathrm{keV}$. In the dual-phase mode used in this study, where the liquid level lies below the top PMT, some of the scintillation light is refracted and reflected at the liquid surface. Though some of the reflected photons may be detected by the bottom PMT, roughly $35 \%$ are lost. The result is a significantly larger S1 signal in the bottom PMT compared to the top (70\% on bottom, $30 \%$ on top), and an overall reduced LY as compared with the value taken in singlephase mode. We measure the dual-phase ${ }^{57} \mathrm{Co}$ zero-field LY to be $6.38 \pm 0.05$ (stat) \pm 0.36 (sys) $\mathrm{pe} / \mathrm{keV}$, with $11.5 \%$ resolution $(\sigma / \mu)$. The systematic uncertainty is taken from the fluctuations of this LY throughout the run, and the statistical uncertainty is the combination of fit uncertainties from each ${ }^{57} \mathrm{Co}$ zero-field data set. The spectrum obtained from one ${ }^{57}$ Co calibration is shown in Fig. 3.

The PMTs were produced with photocathodes specifically designed to have their peak quantum efficiency $(\sim 25 \%$ bottom, $\sim 35 \%$ top) at $178 \mathrm{~nm}$, the wavelength of Xe scintillation light. ${ }^{26}$ Negative high voltage of approximately $-900 \mathrm{~V}$, supplied by an NHQ $225 \mathrm{M}$ high voltage module, is distributed to the photocathodes and 12 dynodes by voltage dividers made in-house on a PTFE substrate with a total impedance of $\sim 140 \mathrm{M} \Omega$. Each PMT is calibrated in situ by a pulsed blue light-emitting diode, periodically and before each measurement, to obtain the single pe response, and hence the PMT gain $\left(\sim 4 \times 10^{6}\right.$ at these voltages). The PMT signals are fed to an external fast voltage amplifier (Phillips 777) having two outputs: one output is connected directly to the analog-to-digital converter (Acqiris model DC436 100 $\mathrm{MS} / \mathrm{s}$ ), while the other is fed to the triggering system. The triggering system is set to discriminate each PMT individually at $\sim 1.5$ pe, requiring coincidence between the two PMT

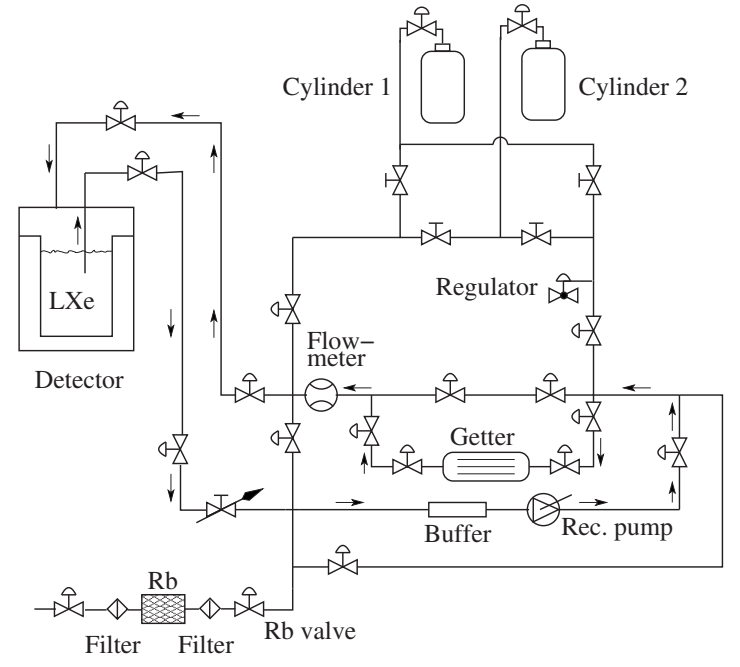

FIG. 4. Schematic of the gas system. The recirculation pump draws liquid up through the recirculation siphon tube in the LXe chamber where it is vaporized while leaving the cryostat space, passed through a getter for purification, and the recondensed inside the chamber. The zeolite beads containing ${ }^{83} \mathrm{Rb}$ are located inside the chamber labeled " $\mathrm{Rb}$ " and exposure to the gas system is controlled by the valve labeled "Rb valve."

channels. The efficiency of this trigger, determined by Monte Carlo method, is $50 \%$ at $1.6 \mathrm{keV}$, rising to $95 \%$ at $4 \mathrm{keV}$, and $99 \%$ at $5.4 \mathrm{keV}$, well below the $32.1 \mathrm{keV}$ events that provide the trigger in a ${ }^{83 \mathrm{~m}} \mathrm{Kr}$ decay. In the offline signal processing, the pulse areas are converted to a value in number of pe by using the measured PMT gains.

In order to maintain the purity of LXe at a level sufficient for optimal light and charge collection, the xenon is constantly purified with a hot metal getter, SAES Mono Torr model PS3MT3R1. The purification is handled by the gas system shown schematically in Fig. 4. Liquid is drawn up from the detector by a closed diaphragm recirculation pump, Enomoto MX-808ST-S, where it is vaporized upon leaving the cryostat. After passing through the pump, it passes through the getter and back into the Xürich detector where it is recondensed. In this way, the xenon is constantly purified in a closed recirculation loop.

${ }^{83 \mathrm{~m}} \mathrm{Kr}$ is introduced into the flow of the closed recirculation circuit by means of a single port with a valve. The zeolite beads containing the ${ }^{83} \mathrm{Rb}$ reside in a small chamber filled with the same xenon gas that flows in the gas system. Gaseous ${ }^{83 \mathrm{~m}} \mathrm{Kr}$ emanating from the ${ }^{83} \mathrm{Rb}$ decay may then diffuse into the recirculation circuit, its introduction being easily controlled by either opening or closing the valve at the port, denoted as the $\mathrm{Rb}$ valve. Due to the rather long half-life of ${ }^{83} \mathrm{Rb}$ (86.2 days), it is imperative that no trace of this mother radionuclide enters the system if it is to be used in a low background experiment. Rb might potentially enter the system by one of two ways: as a vapor, which is very unlikely since its volatility under common laboratory temperatures even under vacuum is not significant, or as an aerosol formed from small particles of the zeolite itself. Aerosol breakthrough is not entirely excluded, and therefore a $0.5 \mu \mathrm{m}$ aerosol filter is placed between the $\mathrm{Rb}$ chamber and the $\mathrm{Rb}$ valve in order to prevent any ${ }^{83} \mathrm{Rb}$ from entering the recirculation loop. Measurements done to assess the level of 

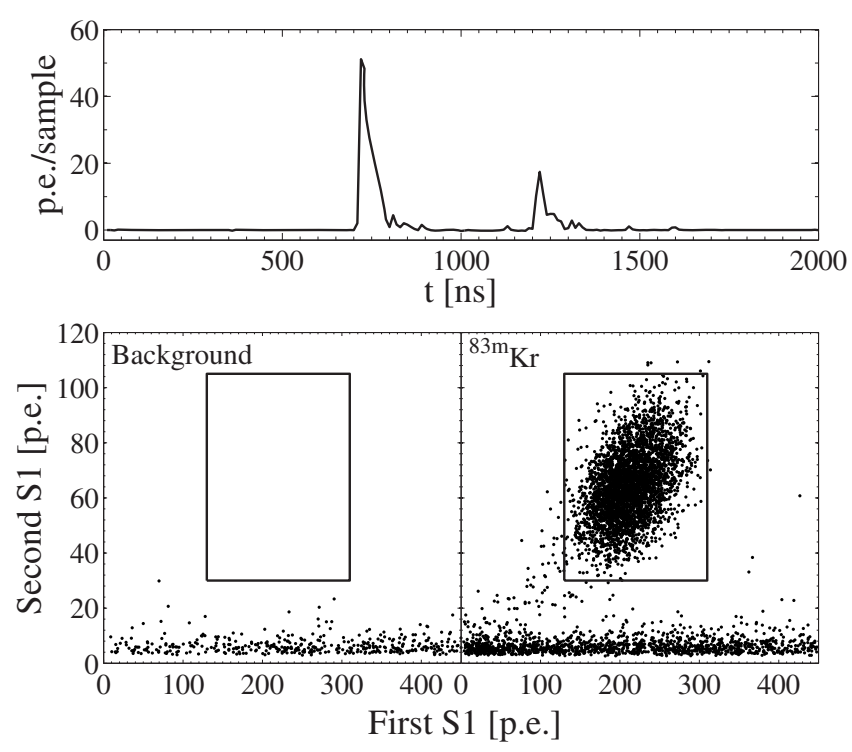

FIG. 5. (Top) PMT output from a ${ }^{83 \mathrm{~m}} \mathrm{Kr}$ decay. In this double pulse of primary scintillation light (S1), the first pulse corresponds to the $32.1 \mathrm{keV}$ transition with the second pulse resulting from the $9.4 \mathrm{keV}$ transition. (Bottom) The area of the first S1 pulse vs the area of the second, for events showing this characteristic two-pulse structure. Shown are distributions taken before $\mathrm{Rb}$ exposure ("background") and during $\mathrm{Rb}$ exposure $\left({ }^{83 \mathrm{~m}} \mathrm{Kr}\right)$, demonstrating that the population of ${ }^{83 \mathrm{~m}} \mathrm{Kr}$ decays is clearly separated from background events. The box represents the energy cuts used as the ${ }^{83 \mathrm{~m}} \mathrm{Kr}$ acceptance window.

${ }^{83} \mathrm{Rb}$ introduced in the system are discussed in Secs. III and IV.

\section{ANALYSIS AND RESULTS}

Once the ${ }^{83 \mathrm{~m}} \mathrm{Kr}$ has entered the LXe, a $32.1 \mathrm{keV}$ transition might occur in the active region, which will then be followed by the $9.4 \mathrm{keV}$ transition. $\mathrm{A}^{83 \mathrm{~m}} \mathrm{Kr}$ decay is, therefore, indicated by two S1 pulses whose separation in time is characterized by a decaying exponential with $t_{1 / 2}=154 \mathrm{~ns}$. Some of these transitions will occur too close in time to be resolved separately, giving a single $41.5 \mathrm{keV}$ pulse; however, the strength of this signal is well below the background level in the Xürich detector. On the other hand, many of the ${ }^{83 \mathrm{~m}} \mathrm{Kr}$ decays have a double $\mathrm{S} 1$ structure, while only a small fraction of non- ${ }^{83 \mathrm{~m}} \mathrm{Kr}$ decay events share this feature. An example of the PMT response from a ${ }^{83 \mathrm{~m}} \mathrm{Kr}$ decay is seen in Fig. 5 (top).

The events with such a double S1 structure are shown from one data set in Fig. 5 (bottom), with the area of the first pulse plotted versus the area of the second pulse. In this space, it is evident that the ${ }^{83 \mathrm{~m}} \mathrm{Kr}$ decays form a population of events that is clearly separated from background. The box indicates the energy cuts for first and second S1 pulses used to identify ${ }^{83 \mathrm{~m}} \mathrm{Kr}$ decays; before opening the $\mathrm{Rb}$ valve, background data show no events within this box. After the Rb valve has been opened, the rate of ${ }^{83 \mathrm{~m}} \mathrm{Kr}$ decays in the total LXe volume increases to the $20 \mathrm{~Bq}$ level in roughly $10 \mathrm{~h}$. In order to further check that these are indeed ${ }^{83 \mathrm{~m}} \mathrm{Kr}$ decays, the distribution of S1 delay times (i.e., the time between the first and second $\mathrm{S} 1$ pulses), $\Delta t_{\mathrm{S} 1}$, of events within the box of Fig. 5 (bottom) is fit with a decaying exponential. The result of the fit, shown in Fig. 6 (top), gives $t_{1 / 2}=156 \pm 5$ ns, consis-
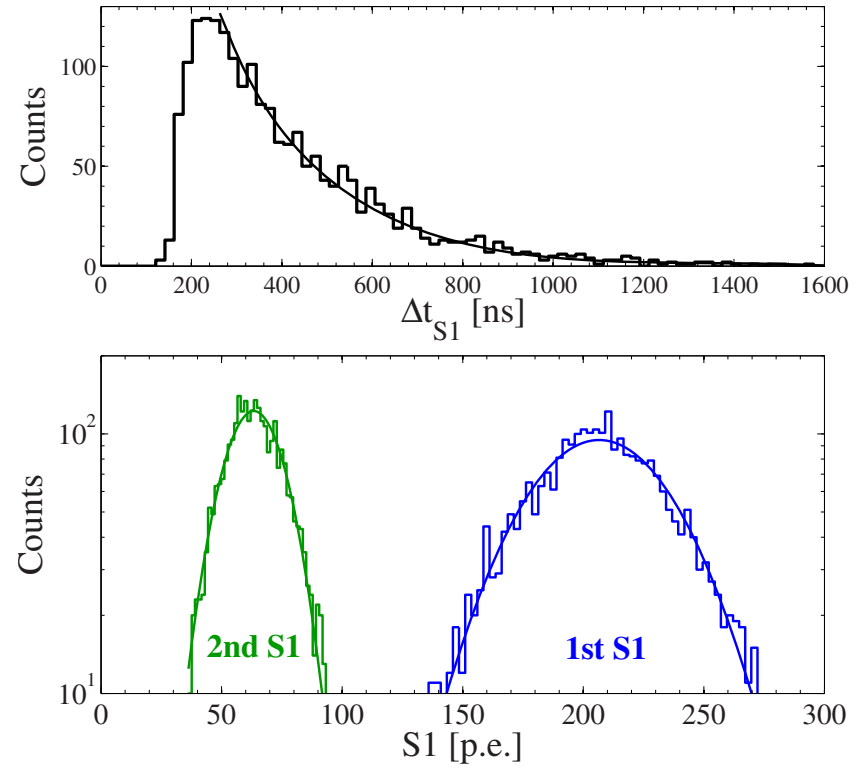

FIG. 6. (Color online) (Top) The distribution of delay times between first and second $\mathrm{S} 1$ pulses for events in the ${ }^{83 \mathrm{~m}} \mathrm{Kr}$ acceptance window. An exponential fit to the distribution gives a half-life of $156 \pm 5 \mathrm{~ns}$, consistent with the published value of $154 \mathrm{~ns}$. (Bottom) Spectra from the two ${ }^{83 \mathrm{~m}} \mathrm{Kr}$ transitions, summed over all runs taken at zero field.

tent with the published value of $154.4 \pm 1.1 \mathrm{~ns}^{21}$ This excellent agreement validates the claim that these events are indeed caused by ${ }^{83 \mathrm{~m}} \mathrm{Kr}$ decays.

Due to the shaping of the PMT signals by the various data acquisition (DAQ) components, multiple $\mathrm{S} 1$ pulses that are delayed by less than $\sim 100$ ns cannot be separately resolved. Additionally, the signal is required to be "clean" (i.e., flat baseline) two samples before and after the pulse, in order to register as a positive $\mathrm{S} 1$ identification during the offline processing of the data. This makes the efficiency for detecting multiple $\mathrm{S} 1$ pulses less than unity for $\Delta t_{\mathrm{S} 1}<250 \mathrm{~ns}$, as is obvious from Fig. 6 (top). Therefore, the double S1 selection cut detects ${ }^{83 \mathrm{~m}} \mathrm{Kr}$ decays with an efficiency of approximately $32 \%$ under these conditions.

The spectra, in pe, obtained at zero field from the two transitions of ${ }^{83 \mathrm{~m}} \mathrm{Kr}$ are displayed in Fig. 6 (bottom). A Gaussian function is fit to each spectrum that is used to determine the LY and energy resolution, shown in Table I. As mentioned in Sec. I, ${ }^{57} \mathrm{Co}$ emits primarily $122 \mathrm{keV} \gamma$ rays. However, there is a small additional contribution from 136 $\mathrm{keV}$. The two lines, however, are not distinguishable from one another due to the detector's energy resolution and instead give a single peak, whose weighted average energy is $123.6 \mathrm{keV}$. The measurements suggest a rise in the LY at lower energies, consistent with behavior previously observed in LXe (Ref. 27) and also in the XENON10 detector. ${ }^{28}$ The peak resolutions $(\sigma / \mu)$ are also shown at zero field.

As mentioned in Sec. II, most LXe detectors use an applied external electric field in order to collect electrons emitted from the interaction site. As the applied field is increased, more and more electrons leave the interaction, suppressing the recombination process that contributes photons to the scintillation signal. The result is that both the scintillation and ionization responses vary strongly with applied field, 
TABLE I. The measured zero-field LY, peak resolution (Res.), and field dependence fit parameters $\left(a_{i}\right)$. The row following $41.5 \mathrm{keV}$ gives the charge collection of the summed signal. Uncertainties shown in LY are statistical only because these two peaks are taken from identical events, their systematic uncertainties are highly correlated, and hence do not affect the significance of the relative rise in LY.

\begin{tabular}{rccccc}
\hline \hline \multicolumn{1}{c}{$\begin{array}{c}E \\
(\mathrm{keV})\end{array}$} & $\begin{array}{c}\mathrm{LY} \\
(\mathrm{pe} / \mathrm{keV})\end{array}$ & $\begin{array}{c}\text { Res. } \\
(\sigma / \mu)\end{array}$ & $a_{1}$ & $\begin{array}{c}a_{2} \\
\left(10^{-4} \mathrm{~cm} / \mathrm{V}\right)\end{array}$ & $a_{3}$ \\
\hline 9.4 & $6.74 \pm 0.06$ & $20.0 \%$ & $-0.35 \pm 0.06$ & $6.3 \pm 3.0$ & 1 \\
32.1 & $6.43 \pm 0.04$ & $14.4 \%$ & $-0.55 \pm 0.03$ & $8.9 \pm 1.6$ & 1 \\
41.5 & $\ldots$ & $\cdots$ & $0.406 \pm 0.006$ & $17 \pm 2$ & $0.074 \pm 0.012$ \\
123.6 & $6.38 \pm 0.05$ & $11.5 \%$ & $-0.679 \pm 0.007$ & $12.6 \pm 0.5$ & 1 \\
\hline \hline
\end{tabular}

with the two signals exhibiting anticorrelation. It is then crucial that the scintillation dependence on the applied field, called field quenching, be known quantitatively for any calibration sources. Figure 7 shows the LY as a function of the applied field, normalized to the zero field value, of the two ${ }^{83 \mathrm{~m}} \mathrm{Kr}$ transitions and the ${ }^{57} \mathrm{Co}$ line. The uncertainty in the LY is dominated by a $2 \%$ systematic uncertainty taken from the measured fluctuations in the PMT gain over the duration of the run. The horizontal positions are determined by electrostatic field simulations of the detector in each voltage configuration used; horizontal uncertainties are the $1-\sigma$ variation in the field over the active volume. The simulations were carried out using the COMSOL simulation package (commercially available), ${ }^{29}$ and verified with software written in house.

The time scale of the ionization signal 1-2 $\mu$ s does not permit the two ${ }^{83 \mathrm{~m}} \mathrm{Kr}$ transitions to be resolved separately, and instead the S2 signal contains the combination of charge emitted from both decays. This $41.5 \mathrm{keV}$ summed-signal ionization yield is also shown in Fig. 7 normalized to $Q_{0}$, the theoretical total amount of initial charge produced prior to electron-ion recombination. This value is determined by plot-

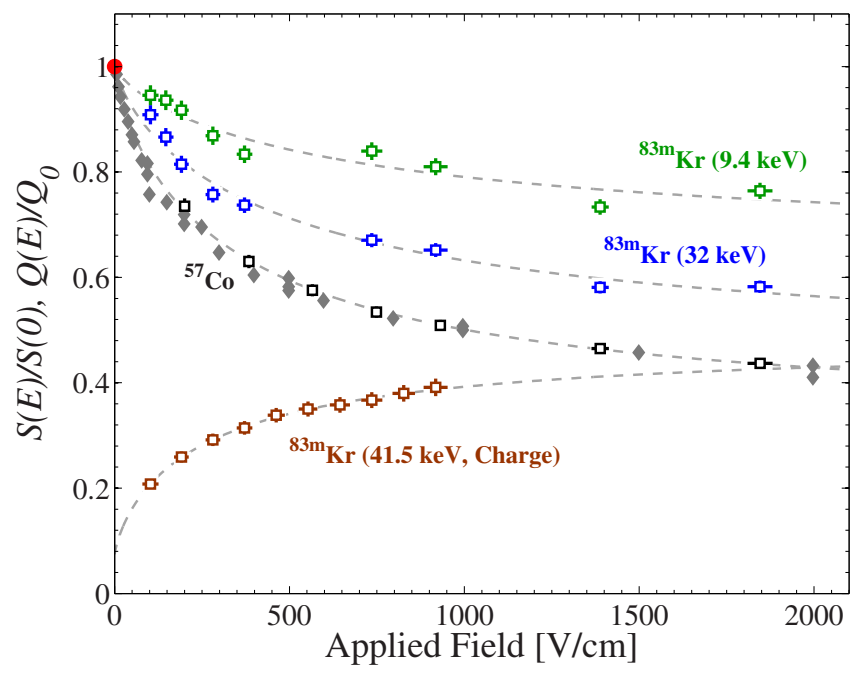

FIG. 7. (Color online) Field quenching, defined as the LY of a spectral line divided by the LY obtained at zero field, or $S(E) / S(0)$. Data collected from ${ }^{57} \mathrm{Co}$ (open black squares) are consistent with those reported in Ref. 31 (solid gray diamonds). Dashed lines correspond to a fit parametrization described in the text. Also shown is the field-dependent charge collection of the combination of both ${ }^{83 \mathrm{~m}} \mathrm{Kr}$ transitions, $Q(E) / Q_{0}$; the two transitions occur too close in time for their ionization signals to be individually resolved. ting the $\mathrm{S} 1$ peak positions versus the $\mathrm{S} 2$ peak positions from data taken at various applied fields, shown in Fig. 8. As S1 and S2 are anticorrelated, these data lie along a line having negative slope, with the line's intercepts representing the total number of quanta, ions plus excitons $\left(N_{\mathrm{ion}}+N_{\mathrm{ex}}\right)$. For electronic recoils, the ratio of excitons to ions, $N_{\mathrm{ex}} / N_{\text {ion }}$, is taken to be $0.06,{ }^{30}$ and hence $Q_{0}$ is $94.3 \%$ the value of the $\mathrm{S} 2$ intercept. The horizontal positions and error bars are determined in the same manner as those of the scintillation yield measurements, while the vertical error bars are instead dominated by the statistical errors in the peak fits and the uncertainty in $Q_{0}$.

The data are fit with a three-parameter function based on the Thomas-Imel box model for electron-ion recombination, ${ }^{32}$ given by

$$
\frac{S(E)}{S(0)}, \frac{Q(E)}{Q_{0}}=a_{1} a_{2} E \ln \left(1+\frac{1}{a_{2} E}\right)+a_{3},
$$

where $E$ is the electric field strength, and $S$ and $Q$ are the scintillation and ionization yields, respectively. This model is used only to provide a convenient parametrization of the data, and not to infer fundamental LXe physical properties from the results of the fits. The $a_{i}$ are the parameters of the

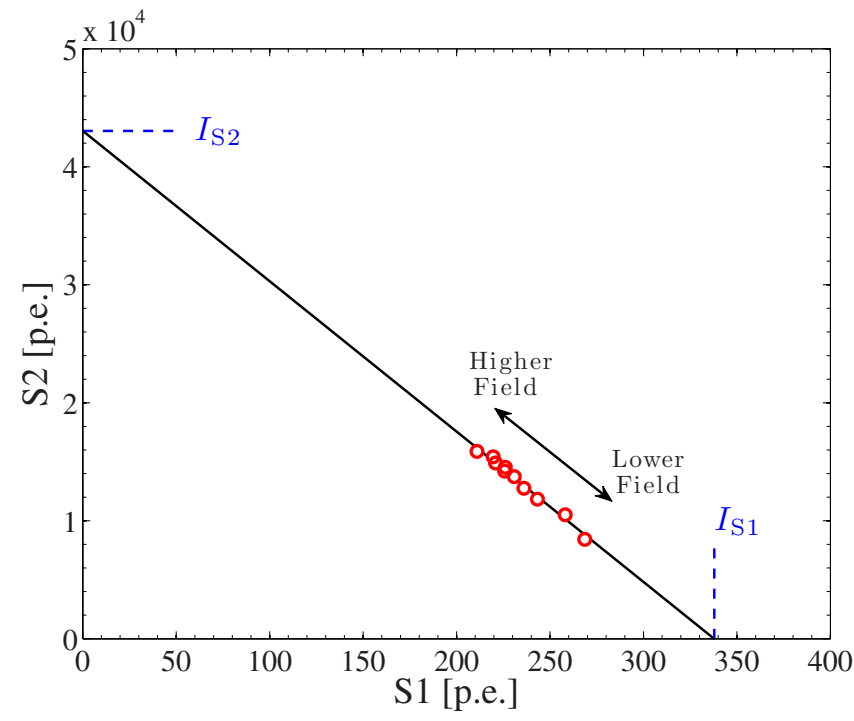

FIG. 8. (Color online) The peak position in S2 vs S1 space for the $41.5 \mathrm{keV}$ emission of ${ }^{83 \mathrm{~m}} \mathrm{Kr}$. The data are taken from applied fields ranging from 100 $\mathrm{V} / \mathrm{cm}$ to $1 \mathrm{kV} / \mathrm{cm}$. The line is fit to the data having vertical and horizontal intercepts $I_{\mathrm{S} 2}$ and $I_{\mathrm{S} 1}$, respectively; these intercepts indicate the location of $N_{\text {ion }}+N_{\text {ex }}$. 

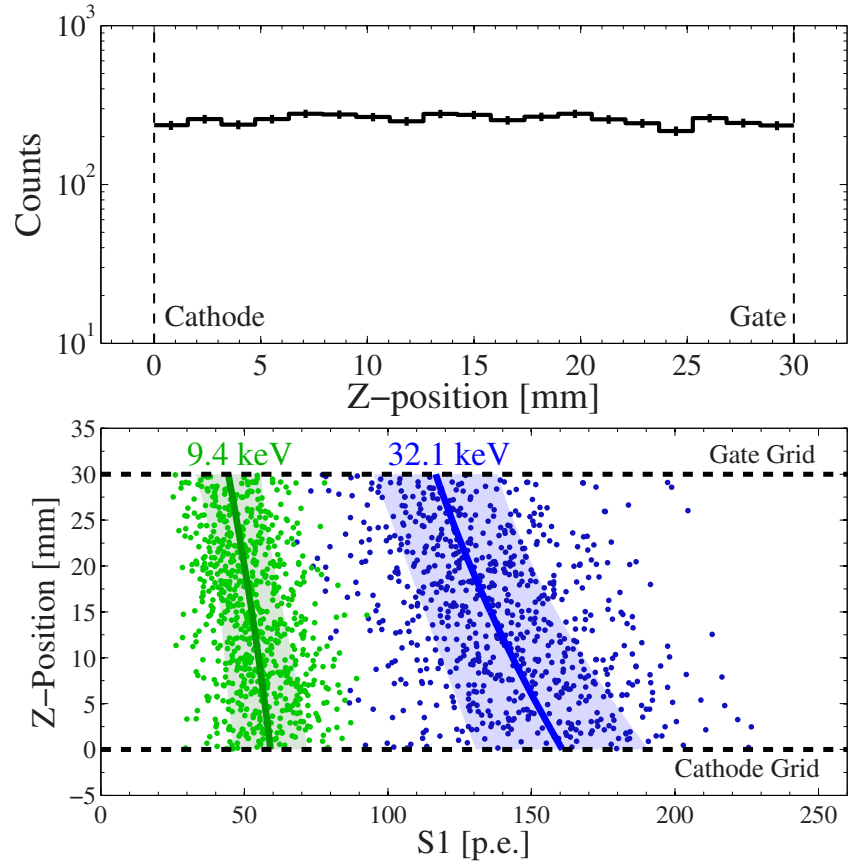

FIG. 9. (Color online) (Top) Rate of ${ }^{83 \mathrm{~m}} \mathrm{Kr}$ decays as a function of $z$ position, indicating a uniform concentration. (Bottom) Measured $z$ dependence on the LY from ${ }^{83 \mathrm{~m}} \mathrm{Kr}$ 's two transitions taken at $1 \mathrm{kV} / \mathrm{cm}$. The solid lines indicated the band centers, with $\pm 1 \sigma$ covered by the shaded areas. Both lines show a LY at the cathode that is a factor of 1.3 larger than at the gate grid.

fit, shown in Table I. Because the scintillation yields are normalized to the value at zero field, $a_{3}$ is unity and the function therefore contains only two free parameters for the scintillation yield data. At decreasing energies, we observe a consistent decrease in the level of field quenching.

The energy of an event can also be measured by counting the total number of quanta, $N_{\text {ion }}+N_{\text {ex }}$. This is called the combined energy scale (CES), and is constructed by forming a linear combination of the scintillation and ionization signals, $\alpha \mathrm{S} 1+\beta \mathrm{S} 2$, such that $n_{\gamma}=\alpha \mathrm{S} 1$ and $n_{e}=\beta \mathrm{S} 2$, where $n_{\gamma}$ and $n_{e}$ are the number of emitted photons and electrons, respectively. The coefficients $\alpha$ and $\beta$ can be found from the plot of S1 versus S2 (Fig. 8), by $\alpha=E /\left(W I_{\mathrm{S} 1}\right)$ and $\beta$ $=E /\left(W I_{\mathrm{S} 2}\right)$, where $E$ is the deposited energy, $W=13.5 \mathrm{eV}$ is the average energy required to produce a single quanta (electron or photon), ${ }^{33}$ and $I_{\mathrm{S} 1(\mathrm{~S} 2)}$ is the $\mathrm{S} 1(\mathrm{~S} 2)$ intercept in units of pe. The CES has the advantage that it is not affected by correlated recombination fluctuations which dominate the S1 resolution over most energies, ${ }^{32}$ and hence gives an energy estimate with better resolution than S1 or S2 alone. For example, the S1-only and S2-only peak resolutions of the 41.5 $\mathrm{keV}$ peak taken at $500 \mathrm{~V} / \mathrm{cm}$ are $14.2 \%$ and $20.1 \%$, respectively, while the resolution of the CES peak at this field is $10.0 \%$.

The delay time between $\mathrm{S} 1$ and S2 gives the drift time of the electrons, and hence the $z$ position of the interaction. One important motivation for using this source is that it should disperse uniformly in the active LXe volume, providing a spatially uniform calibration. The summed $z$-position distribution of ${ }^{83 \mathrm{~m}} \mathrm{Kr}$ events taken at drift fields from $100-1000$ V/cm is shown in Fig. 9 (top). The observed $z$-dependent rate is flat with variations consistent with statistical fluctuations on each bin. With this uniform calibration, the position dependence of the detector's response can be measured and corrected for. Most of the S1 signal is detected by the bottom PMT, and therefore one expects to see a LY that is a monotonically decreasing function of $z$ position (i.e., more light is collected from events occurring close to the bottom PMT than for events close to the top). Figure 9 (bottom) shows the $\mathrm{LY}$ of the ${ }^{83 \mathrm{~m}} \mathrm{Kr}$ decays at all positions along the $z$ axis between the cathode and gate grids for the data run at 1 $\mathrm{kV} / \mathrm{cm}$; solid lines are the band centroids, shaded bands cover $\pm 1 \sigma$. At both energies, the LY at the cathode (bottom of active region) is a factor of 1.3 higher than the LY at the gate grid (top of active region). Data collected at lower applied fields show consistency with this behavior.

Although the ${ }^{83 \mathrm{~m}} \mathrm{Kr}$ decays away in a matter of hours, the ${ }^{83} \mathrm{Rb}$ will live for nearly 1.5 years before decaying below $1 \%$ of the initial activity. If this technique is to be used in low-background experiments, it is then imperative that no ${ }^{83} \mathrm{Rb}$ atoms enter the system, and instead must remain trapped within the zeolite or the filter. In order to test this, the valve to the ${ }^{83} \mathrm{Rb}$ chamber was closed. The rate of ${ }^{83 \mathrm{~m}} \mathrm{Kr}$ decays is expected to decrease exponentially to zero during the following day; however, if ${ }^{83} \mathrm{Rb}$ has entered the system, the rate versus time will behave as an exponential decay with a vertical offset. No such offset was observed in the ${ }^{83 \mathrm{~m}} \mathrm{Kr}$ rate following the closing of the $\mathrm{Rb}$ valve. Indeed, $2.5 \mathrm{~h}$ of data collected one day after closing the $\mathrm{Rb}$ valve resulted in zero observed events. This null observation corresponds to a one-sided Poisson upper limit of $<800 \mu \mathrm{Bq}, 90 \%$ confidence level (C.L.), of residual ${ }^{83 \mathrm{~m}} \mathrm{Kr}$ in the active region.

\section{DISCUSSION}

Testing the low energy response of LXe is generally rather difficult, and therefore ${ }^{83 \mathrm{~m}} \mathrm{Kr}$ provides a unique tool for such measurements. The rate of ${ }^{83 \mathrm{~m}} \mathrm{Kr}$ decays studied in this work is quite low as compared with the rate of background events due to natural radioactivity and cosmic rays, contributing roughly $0.5 \%$ of the total triggers. However, the double S1 structure of these decays and energy cuts used enable their measurement in a virtually background-free regime. It is therefore not necessary to use a low background setup simply to study this weak source. Simultaneous with this work, a demonstration of ${ }^{83 \mathrm{~m}} \mathrm{Kr}$ introduction to a singlephase LXe chamber by a similar technique has been performed by another group. ${ }^{20}$

The LY and energy resolution at low energies are of particular relevance for dark matter direct detection searches. As indicated in Table I, the LY increases at low energies. Although an accurate quantitative understanding of this process is incomplete, the observed behavior can be understood qualitatively in the following manner. The electronic stopping power of electrons in LXe increases at decreasing energies, ${ }^{34}$ and thus the ionization density produced by a recoiling electron increases along the track, with the highest densities concentrated at the track's end. Because of this, the overall ionization density caused by a low energy recoiling electron will be greater than for an electron of higher energy. 
The electrons and ions produced along the track will rapidly recombine and produce scintillation photons as they deexcite to their ground states. The strength of recombination is correlated with the ionization density because the characteristic electron-ion distance is shorter for higher ionization densities. Even at zero applied electric field, not all of the electron-ion pairs produced by a recoiling particle will recombine to give scintillation photons. ${ }^{11}$ It is then expected that the zero field recombination is stronger at lower energies (higher $d E / d x$ ), giving a higher overall LY.

In Ref. $22,4 \mathrm{~h}$ baking at $300{ }^{\circ} \mathrm{C}$ resulted in no observed loss of ${ }^{83} \mathrm{Rb}$. The observation in this study of no ${ }^{83 \mathrm{~m}} \mathrm{Kr}$ decays, one day following the closing of the $\mathrm{Rb}$ valve, sets an upper limit of $800 \mu \mathrm{Bq}$ of residual ${ }^{83 \mathrm{~m}} \mathrm{Kr}$ inside the active region of the Xürich detector. Prior to this, the $\mathrm{Rb}$ valve had been opened for a total of $150 \mathrm{~h}$ during the run. The risk of $\mathrm{Rb}$ contamination increases with the amount of time that the valve is opened, and so this upper limit can be normalized to exposure time $(150 \mathrm{~h})$. Moreover, since the source is exposed to the gas system (and not the detector), the total activity in the LXe chamber should be independent of the detector size, and should instead depend on only flow rate and method of deployment. The limit of $800 \mu \mathrm{Bq}$ in the active region $(0.08$ $\mathrm{kg}$ ) can be scaled to the total amount of LXe in the chamber $(1.76 \mathrm{~kg})$, and normalized to the exposure, to give $<120 \mu \mathrm{Bq} / \mathrm{h}$ of residual ${ }^{83 \mathrm{~m}} \mathrm{Kr}$ in the whole liquid volume (assuming that the ${ }^{83 \mathrm{~m}} \mathrm{Kr}$ concentration outside the active region is uniform and equal to the concentration inside the active region). The branching ratio of ${ }^{83} \mathrm{Rb}$ to ${ }^{83 \mathrm{~m}} \mathrm{Kr}$ is $75 \%$, which means that this limit on residual ${ }^{83 \mathrm{~m}} \mathrm{Kr}$ is a limit of $<160 \mu \mathrm{Bq} / \mathrm{h}$ of residual ${ }^{83} \mathrm{Rb}$ contamination.

To understand how this upper limit would affect an actual dark matter search, a $300 \mathrm{~kg}$ detector with $100 \mathrm{~kg}$ fiducial mass is taken as an example. A detector of this size is typical of the proposed next generation of LXe dark matter searches. ${ }^{35,36}$ An exposure to ${ }^{83} \mathrm{Rb}$ of $10 \mathrm{~h}$ would be sufficient, under these conditions, to provide adequate statistics for such a calibration $(\sim 1000 \mathrm{Kr}$ events $/ \mathrm{kg})$. Our upper limit of ${ }^{83} \mathrm{Rb}$ contamination translates to a residual rate of $<0.46$ events $/ \mathrm{kg}^{-1} / \mathrm{day}^{-1}$ in this $300 \mathrm{~kg}$. Even if this amount of ${ }^{83} \mathrm{Rb}$ was present in the system, the vast majority of decays would not introduce dangerous backgrounds. In order for a background event to be "dangerous" (i.e., appear in the WIMP signal acceptance window), it must have two features: (1) it must produce a single scatter event and (2) the event must deposit a small amount of energy that is within the WIMP search energy window. An additional feature that dual-phase LXe TPCs such as Xürich have is the ability to reject electronic recoils on an event-by-event basis at the level of $\sim 99.9 \%$ based on the ratio S2/S1. ${ }^{37}$ However, statistical fluctuations can cause a small fraction of electronic recoil events to yield an S2/S1 ratio similar to values characteristic of a nuclear recoil from WIMPs, and thus the overall background level must be minimized as much as possible. Any ${ }^{83 \mathrm{~m}} \mathrm{Kr}$ decays in the active volume would not present a problem because they would either have a double S1 structure (and could be vetoed on that basis), or would give 41.5 $\mathrm{keV}$, outside of the WIMP search region. The only possibility for a dangerous background is from one of the $\gamma$ rays pro- duced as the initial excited ${ }^{83} \mathrm{Kr}$ decays to the metastable state. These $\gamma$ rays are mostly emitted in the range of 500$600 \mathrm{keV}$; again, to be dangerous they are required to single scatter in the fiducial region, which is highly unlikely given their 3-4 cm attenuation length. With ${ }^{83} \mathrm{Rb}$ contamination at the level of our upper limit, Monte Carlo simulations indicate that 0.46 decays $/ \mathrm{kg}$ day would contribute less than $67 \mu$ DRU of single scatters in the WIMP search energy region $\left(1 \mathrm{DRU} \equiv 1\right.$ event kg${ }^{-1} \mathrm{day}^{-1} \mathrm{keV}^{-1}$ ). The projected $\gamma$ background rate in Refs. 35 and 36 due to natural radioactivity in the detector materials alone is roughly $1 \mathrm{mDRU}$, fully 15 times greater than our upper limit on the ${ }^{83} \mathrm{Rb}$ background.

After a calibration with ${ }^{83 \mathrm{~m}} \mathrm{Kr}$, the stable ${ }^{83} \mathrm{Kr}$ will remain in the system indefinitely unless some action is taken to specifically remove it. However, the amount of $\mathrm{Kr}$ remaining from a $10 \mathrm{~h}$ exposure as described above will be miniscule; less than $10^{6}$ atoms total, which corresponds to a concentration of roughly one part in $10^{21}$ for $300 \mathrm{~kg}$ of Xe. Even if this remaining concentration was higher, $\mathrm{Kr}$ will not adversely affect detector functions. The transport of electrons through the Xe will not be diminished since $\mathrm{Kr}$ is chemically similar to Xe. Additionally, $\mathrm{Kr}$ does not absorb Xe scintillation light $^{38}$ and therefore will not impede light collection.

In addition to ${ }^{83} \mathrm{Rb}$ contamination, water and oxygen trapped in the zeolite might also enter the system. While these elements do not pose a problem in the context of radioactive backgrounds, they could affect the charge collection and LY. Before the Rb valve was initially opened, the $\mathrm{Rb}$ chamber was evacuated to the level of $10^{-6}$ mbar with a turbomolecular pump at room temperature. The $\mathrm{Rb}$ valve was then opened continuously for approximately four days, following which diminished charge collection was observed. The $\mathrm{Rb}$ valve was then closed and the purification system allowed time to restore the LXe purity to a level adequate for negligible charge loss. In subsequent measurements, the $\mathrm{Rb}$ valve was toggled in cycles of $20 \mathrm{~h}$ open, $4 \mathrm{~h}$ closed, with charge collection periodically monitored; no charge loss was measured under these conditions. It is likely that the impurity content in the zeolite was depleted in the initial four days of exposure, and had left the system by the time the cycles of $20 \mathrm{~h}$ exposure began. In a subsequent run, the $\mathrm{Rb}$ chamber was baked for $24 \mathrm{~h}$ at $120{ }^{\circ} \mathrm{C}$ prior to exposure, following which no effect on the electron lifetime was observed. At no time was any effect on the light collection seen.

The Xürich detector will be used in the future to study light and charge collection in LXe in response to nuclear recoils, scintillation pulse shape studies, and in studies related to research and development leading to a ton-scale LXe dark matter search.

\section{SUMMARY}

This work presents a method to utilize the radioactive source ${ }^{83 \mathrm{~m}} \mathrm{Kr}$ in a LXe detector. The two transitions of the ${ }^{83 \mathrm{~m}} \mathrm{Kr}$ decay produce two primary scintillation pulses, which allow these events to be clearly separated from background. Additionally, the dual-phase detector used to study this source shows a spatially uniform distribution of decays, vali- 
dating one of the main motivations for studying this source. The data collected with this source show an increase in the LY at decreasing energies, indicating a corresponding increasing level of zero-field electron-ion recombination. A corresponding weaker field dependence of the LY at decreasing energies is also observed. An alternative energy scale based on the combination of scintillation and ionization signals was shown to provide superior energy resolution at 41.5 $\mathrm{keV}$ compared with scintillation or ionization alone.

The technique used to introduce ${ }^{83 \mathrm{~m}} \mathrm{Kr}$ involves exposing the system to ${ }^{83} \mathrm{Rb}$, the presence of which in a lowbackground experiment could yield serious problems if contamination occurs. This measurement provides an upper limit on the level of ${ }^{83} \mathrm{Rb}$ contamination introduced to the system of $<160 \mu \mathrm{Bq} / \mathrm{h}$ exposure. In the context of the next generation of LXe dark matter direct detection searches, this upper limit translates to a maximum introduced background of $67 \mu \mathrm{DRU}$, far below the $1 \mathrm{mDRU}$ of intrinsic backgrounds.

\section{ACKNOWLEDGMENTS}

The authors wish to thank the machine shop at the Universität Zürich for valuable help in fabrication of the Xürich TPC, and the machine shop at the University of Florida for construction of the cryostat. The work reported here was supported by the Universität Zürich, by the Swiss National Foundation Grant No. 20-118119, and by the Volkswagen Foundation. T.M.U. acknowledges the Alexander von Humboldt Foundation for support through the Feodor Lynen scholarship. Further support was provided by the Ministry of Education, Youth and Sports of the Czech Republic, under Contract Nos. LA318 and LC07050.

${ }^{1}$ UKDM Collaboration, Astropart. Phys. 23, 444 (2005).

${ }^{2}$ UKDM Collaboration, Astropart. Phys. 28, 287 (2007).

${ }^{3}$ UKDM Collaboration, Phys. Rev. D 80, 052010 (2009).

${ }^{4}$ XENON10 Collaboration, Phys. Rev. Lett. 100, 021303 (2008)

${ }^{5}$ XENON10 Collaboration, Phys. Rev. Lett. 101, 091301 (2008).

${ }^{6}$ EXO Collaboration, Nucl. Instrum. Methods Phys. Res. A 578, 409 (2007).

${ }^{7}$ A. Papa, Nuovo Cimento Soc. Ital. Fis., B 123B, 860 (2008).

${ }^{8}$ K. Giboni, E. Aprile, T, Doke, S. Suzuki, L. M. P. Fernandes, J. A. M. Lopes, and J. M. F. dos Santos, JINST 2, 10001 (2007).
${ }^{9}$ M. I. Lopes, V. Chepel, V. Solovov, R. Ferreira-Marques, and A. J. P. L. Policarpo, Proceedings of the Eighth International Conference on Calorimetry in High-Energy Physics (CALOR 99), Lisbon, Portugal, 13-19 June 1999.

${ }^{10}$ L. S. Miller, S. Howe, and W. E. Spear, Phys. Rev. 166, 871 (1968).

${ }^{11}$ T. Doke, A. Hitachi, J. Kikuchi, K. Masuda, H. Okada, and E. Shibamura, Jpn. J. Appl. Phys. 41, 1538 (2002).

${ }^{12}$ G. Bertone, D. Hooper, and J. Silk, Phys. Rep. 405, 279 (2005)

${ }^{13}$ G. Jungman, M. Kamionkowski, and K. Griest, Phys. Rep. 267, 195 (1996).

${ }^{14}$ J. D. Lewin and P. F. Smith, Astropart. Phys. 6, 87 (1996).

${ }^{15}$ K. Ni, T. M. Wongjirad, L. Kastens, A. Monzar, and D. N. McKinsey, Nucl. Instrum. Methods Phys. Res. A 582, 569 (2007).

${ }^{16}$ D. McKinsey, April 2008 DUSEL S4/ISE Workshop, 2008, http:// neutrino.lbl.gov/ dusel/HomestakeWorkshopApril2008/Physics/.

${ }^{17}$ ALEPH Collaboration, Nucl. Instrum. Methods Phys. Res. A 294, 121 (1990).

${ }^{18}$ DELPHI Collaboration, IEEE Trans. Nucl. Sci. 42, 491 (1995).

${ }^{19}$ KATRIN Collaboration, KATRIN Report No. FZKA-7090, 2004.

${ }^{20}$ L. W. Kastens, S. B. Cahn, A. Manzur, and D. N. McKinsey, Phys. Rev. C 80, 045809 (2009).

${ }^{21} \mathrm{~S}$. Chu, L. Ekström, and R. Firestone, WWW Table of Radioactive Isotopes (Lawrence Berkeley National Laboratory, Berkeley, CA, 1999).

${ }^{22}$ D. Vénos, A. Spalek, O. Lebeda, and M. Fiser, Appl. Radiat. Isot. 63, 323 (2005).

${ }^{23}$ See: http://www.hamamatsu.com/ for Hamamatsu Photonics.

${ }^{24}$ A. I. Bolozdynya, Nucl. Instrum. Methods Phys. Res. A 422, 314 (1999).

${ }^{25}$ M. Yamashita, T. Doke, J. Kikuchi, and S. Suzuki, Astropart. Phys. 20, 79 (2003).

${ }^{26}$ O. Cheshnovsky, B. Raz, and J. Jortner, Chem. Phys. Lett. 15, 475 (1972).

${ }^{27}$ M. Yamashita, T. Doke, K. Kawasaki, J. Kikuchi, and S. Suzuki, Nucl. Instrum. Methods Phys. Res. A 535, 692 (2004).

${ }^{28} \mathrm{P}$. Sorensen, Ph.D. thesis, Brown University, 2008.

${ }^{29}$ See: http://www.comsol.com/ for COMSOL MULTiPHYSICS.

${ }^{30}$ T. Takahashi, S. Konno, T. Hamada, M. Miyajima, S. Kubota, A. Nakamoto, A. Hitachi, E. Shibamura, and T. Doke, Phys. Rev. A 12, 1771 (1975).

${ }^{31}$ E. Aprile, C. E. Dahl, L. de Viveiros, R. J. Gaitskell, K. L. Giboni, J. Kwong, P. Majewski, K. Ni, T. Shutt, and M. Yamashita, Phys. Rev. Lett. 97, 081302 (2006).

${ }^{32}$ J. Thomas and D. A. Imel, Phys. Rev. A 36, 614 (1987).

${ }^{33}$ T. Shutt, C. E. Dahl, J. Kwong, A. Bolozdynya, and P. Brusov, Nucl. Instrum. Methods Phys. Res. A 579, 451 (2007).

${ }^{34}$ See: http://physics.nist.gov/PhysRefData/Star/Text/ESTAR.html for ESTAR, stopping powers, and ranges for electrons.

${ }^{35}$ E. Aprile, Proceedings of Identification of Dark Matter, 2008.

${ }^{36} \mathrm{R}$. Gaitskell, Proceedings of Identification of Dark Matter, 2008

${ }^{37}$ XENON10 Collaboration, e-print arXiv:1001.2834 (2010).

${ }^{38}$ G. Baldini, Phys. Rev. 128, 1562 (1962). 